\title{
Improving Atomic-Scale Elemental Mapping Resolution of STEM-EDS through Optimizing Experimental Conditions
}

\author{
Ping $\mathrm{Lu}^{1}$, Renliang Yuan ${ }^{2}$ and Jian Min $\mathrm{Zuo}^{2}$ \\ ${ }^{1}$ Sandia National Laboratories, Albuquerque, NM 87185-1411 USA \\ ${ }^{2}$ Department of Materials Science and Engineering, University of Illinois at Urbana-Champaign, \\ 1304 W Green St, Urbana, IL 61801, USA
}

Atomic-scale elemental mapping in aberration corrected scanning transmission electron microscopy (STEM) using energy-dispersive x-ray spectra (EDS) provides a real-space approach to resolve materials structures [1-3]. The technique offers a benefit of easy interpretation with a one-to-one correspondence between image and structure, and provides a mean to resolve the crystal structure that cannot be resolved otherwise by the traditional imaging or diffraction techniques [1]. However, the technique has a severe shortcoming due to the poor efficiency of $\mathrm{x}$ ray generation and collection, and as a result a typical data collection requires more than a few 100 seconds, subjecting the samples to high irradiation of electrons. Utilizing the method of latticevector translation (or lattice-averaging) [2,3], we have demonstrated that the rapid atomic-scale chemical elemental mapping by STEM-EDS can be obtained with the acquisition time of $\sim 2$ seconds or less. The technique provides averaged atomic-scale elemental maps from an area of few $10 \mathrm{~nm}^{2}$, enabling the elemental mapping of electron-beam sensitive materials as well as the time-resolved chemical mapping [3].

Additional limitation of atomic-scale STEM-EDS that prevents its applications is due to its relatively poorer resolution than that of the STEM image. While the sub-Angström resolution has been routinely obtained in STEM image using high-angle annular dark-field (HAADF) detector, the resolution for the STEM-EDS (defined as the shortest distance between the identical atomic-columns that can be resolved in a projected crystal structure) has been so far limited to around $2 \AA$, even when the STEM-EDS mapping is carried out under similar electron optical conditions [1,2]. In this work, we report our efforts to improve the STEM-EDS resolution by optimizing experimental conditions, and through the studies by modeling. We find that a key parameter for achieving the highest resolution STEM-EDS mapping is the specimen thickness, and it is necessary to be below a certain crystal thickness. Using the $\mathrm{SrTiO}_{3}$ (STO) crystal in [001] projection as an example, we demonstrate that atomic-scale EDS mapping with a resolution approaching to HAADF image can be obtained when the specimen thickness is limited to be less than $20 \mathrm{~nm}$ for $200 \mathrm{keV}$ electrons. This thickness effect can be explained based on the $\mathrm{x}$-ray delocalization in the crystal by using multislice image simulations.

A FEI Titan ${ }^{\mathrm{TM}}$ G2 80-200 STEM with a Cs probe corrector and ChemiSTEM ${ }^{\mathrm{TM}}$ technology (X-FEG ${ }^{\mathrm{TM}}$ and SuperX ${ }^{\mathrm{TM}} \mathrm{EDS}$ with four windowless silicon drift detectors) operated at $200 \mathrm{kV}$ was used in this study. An electron probe of size less than $0.13 \mathrm{~nm}$, convergence angle of 18.1 $\mathrm{mrad}$, and current of $\sim 75 \mathrm{pA}$ was used for data acquisition. HAADF images were recorded under similar optical conditions using an annular detector with a collection range of 60-160 mrad. Figures $1 \mathrm{a}, 1 \mathrm{~b}$, and $1 \mathrm{c}$ show $\mathrm{Sr}(\mathrm{K}$ and $\mathrm{L})$ map, Ti $\mathrm{K}$ map and HAADF image, respectively, obtained simultaneously from a thin STO crystal in [001] direction. The crystal thickness is about $16 \mathrm{~nm}$, which was determined by using position-averaged convergent beam electron diffraction [4]. Under the thin specimen condition, high-quality EDS maps, exhibiting narrow and high peakto-valley $(\mathrm{P} / \mathrm{V})$ ratio peak about the respective atomic columns, can be obtained. Figure 1d shows the 3D surface-plot of Ti K map, showing the narrow Ti peaks with a P/V ratio of greater than 7.0. 
The line-profiles across Ti columns along [100] direction (Fig.1e) show the width of Ti EDS peak match closely with that of HAADF image. The full-width at half-maximum (FWHM) for Ti EDS peak reaches about $0.16 \mathrm{~nm}$, slightly broader than $0.13 \mathrm{~nm}$ for HAADF, which is expected from the effective ionization potentials [5]. Both the higher P/V value (>7.0) and narrower FWHM width (about $0.16 \mathrm{~nm}$ ) achieved under our experimental conditions represent a significant improvement from the current state of atomic-scale EDS mapping [6].

1. Lu, P., Zhou, L., Kramer, M.J., \& Smith, D.J. Sci. Rep. 4, 3945 (2014).

2. Lu, P., Romero, E., Lee, S., MacManus-Driscoll, J. L., \& Jia, Q. Microsc. Microanal 20, 1782-1790 (2014).

3. Lu, P., Yuan, R.L., Ihlefeld, J. F., Spoerke, E.D., Pan, W., \& Zuo, J. M. Nano Letter 16, 2728-2733 (2016).

4. LeBeau, J.M., Findlay, S.D., Allen, L.J., \& Stemmer, S. Ultramicroscopy 110,118 (2010).

5. D’Alfonso, A.J., Freitag, B., Klenov, V. \& Allen, L.J. Phys. Rev. B 81, 100101 (2010).

6. Sandia National Laboratories is a multi-program laboratory managed and operated by Sandia Corporation, a wholly owned subsidiary of Lockheed Martin Corporation, for the US Department of Energy's National Nuclear Security Administration under contract DE-AC0494AL85000.
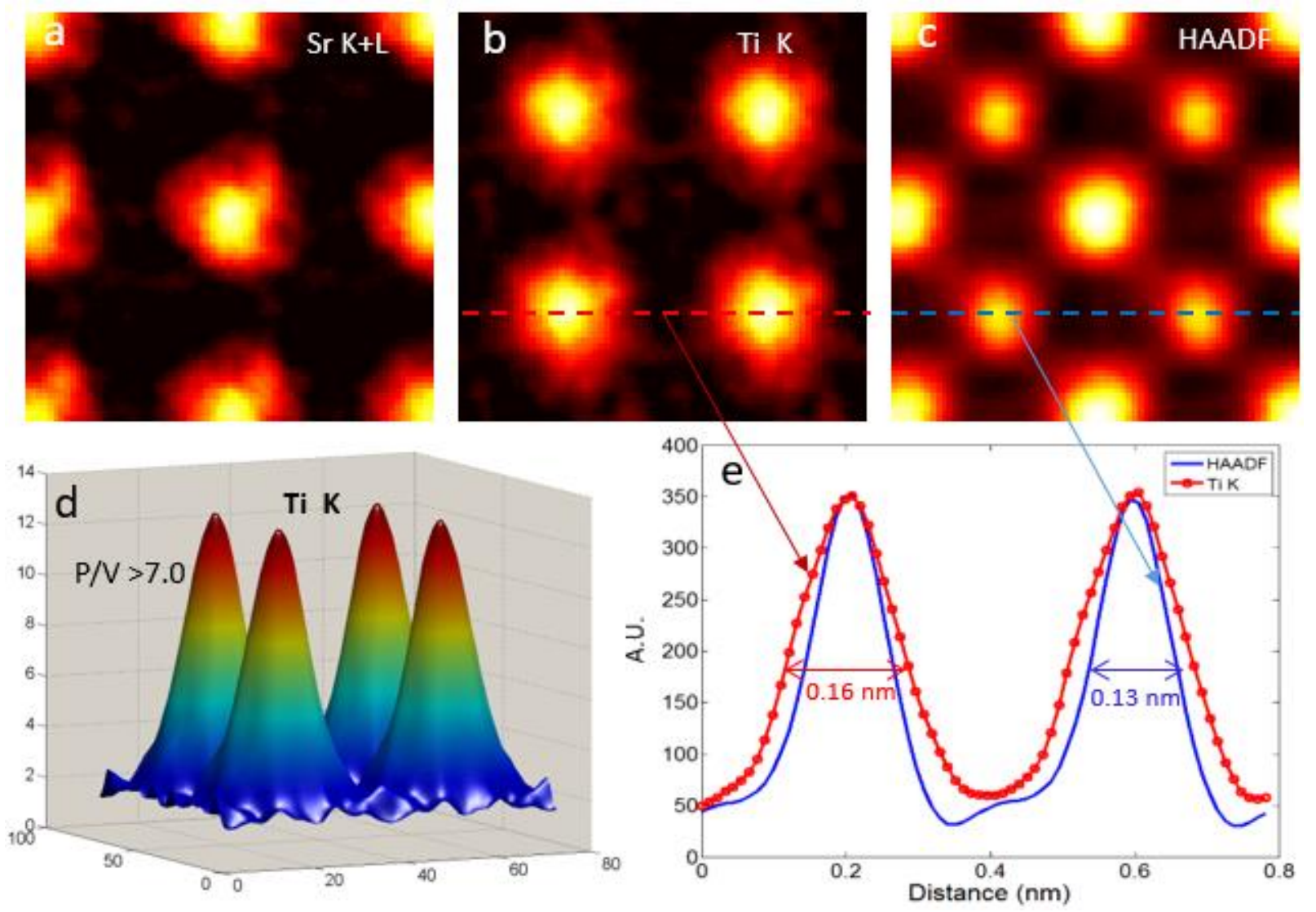

Figure 1. (a) EDS Sr (K+L) map; (b) EDS Ti K map; (c) HAADF image, obtained from a thin STO crystal ( 16nm) in [001] projection; (d) 3D surface-plot of Ti K map; and (e) line-profiles of EDS and HAADF across Ti column along [100] direction. 